\title{
The next chapter in reverse vaccinology
}

\author{
James M Musser \\ Analysis of group A Streptococcus cell-surface proteins yields a new mouse-protective antigen.
}

Vaccine development has emerged as a compelling example of the benefits of genomics. In the last five years, the traditional, linear process of testing antigens one at a time has been revolutionized by genome-scale, parallel strategies for discovering candidate antigens — an approach commonly referred to as 'reverse vaccinology' In this issue, Rodriguez-Ortega et al. ${ }^{2}$ push this approach even further, using a combined biochemical, proteomic, and bioinformatics strategy to identify fragments of proteins displayed on the cell surface of group A Streptococcus (Fig. 1). The result of their research was the discovery of a new protein that protects mice from lethal intranasal challenge with virulent group A Streptococcus. Even the most jaded student of genome-scale analyses should appreciate this work.

Extracellular molecules made by bacterial pathogens have long been a target of vaccine developers. The search for such molecules by reverse vaccinology, which generally involves brute-force cloning, overexpression, purification and animal model testing of many hundreds of recombinant proteins, has resulted in the most remarkable burst of candidate antigen discovery in history. Investigators at Chiron Vaccines have led the way, from their pioneering work on Neisseria meningitidis to a recent tour-de-force analysis of the key neonatal pathogen group B Streptococcus ${ }^{3,4}$. These previous studies were very labor intensive, expensive and relatively slow. Rodriguez-Ortega et al. have achieved a more targeted and efficient strategy by restricting their efforts to proteins on the bacterial cell surface, which are potentially readily accessible to host immune functions such as opsonizing antibodies.

James M. Musser is at the Center for Molecular and Translational Human Infectious Disease Research, The Methodist Hospital Research Institute, The Methodist Hospital, 6565 Fannin Street, Houston, Texas 77030, USA.

e-mail:jmmusser@tmh.tmc.edu
Group A Streptococcus are human specialist pathogens responsible for an estimated 600 million cases of pharyngitis (sore throat) worldwide per year ${ }^{5}$. These bacteria also remain the primary cause of preventable childhood heart disease globally by virtue of their ability to cause rheumatic fever and rheumatic heart disease, two postinfection sequelae. Severe invasive infections caused by group A Streptococcus, such as necrotizing fasciitis, commonly known as the 'flesh-eating' syndrome, have attracted much scientific and lay public attention in recent years. The search for a vaccine has a long and storied history marked by many false leads, blind alleys and abject failures, and despite many decades of research, there is still no licensed human vaccine to protect against group A Streptococcus infections.

Like so many important advances in this field, the work of Rodriguez-Ortega et al. has its origin in experiments performed in Rebecca Lancefield's laboratory at Rockefeller University. Lancefield studied pathogenic streptococci for more than 50 years. Her crucial early work on group A Streptococcus in the 1920s demonstrated that the type-specific antigen of these bacteria was $\mathrm{M}$ protein ${ }^{6}$. This result was extraordinary because the dogma at the time was that type specificity in pathogenic bacteria was due solely to capsular polysaccharides, as shown initially by Oswald T. Avery and Michael Heidelberger. Lancefield ${ }^{7}$ subsequently reported in 1943 that

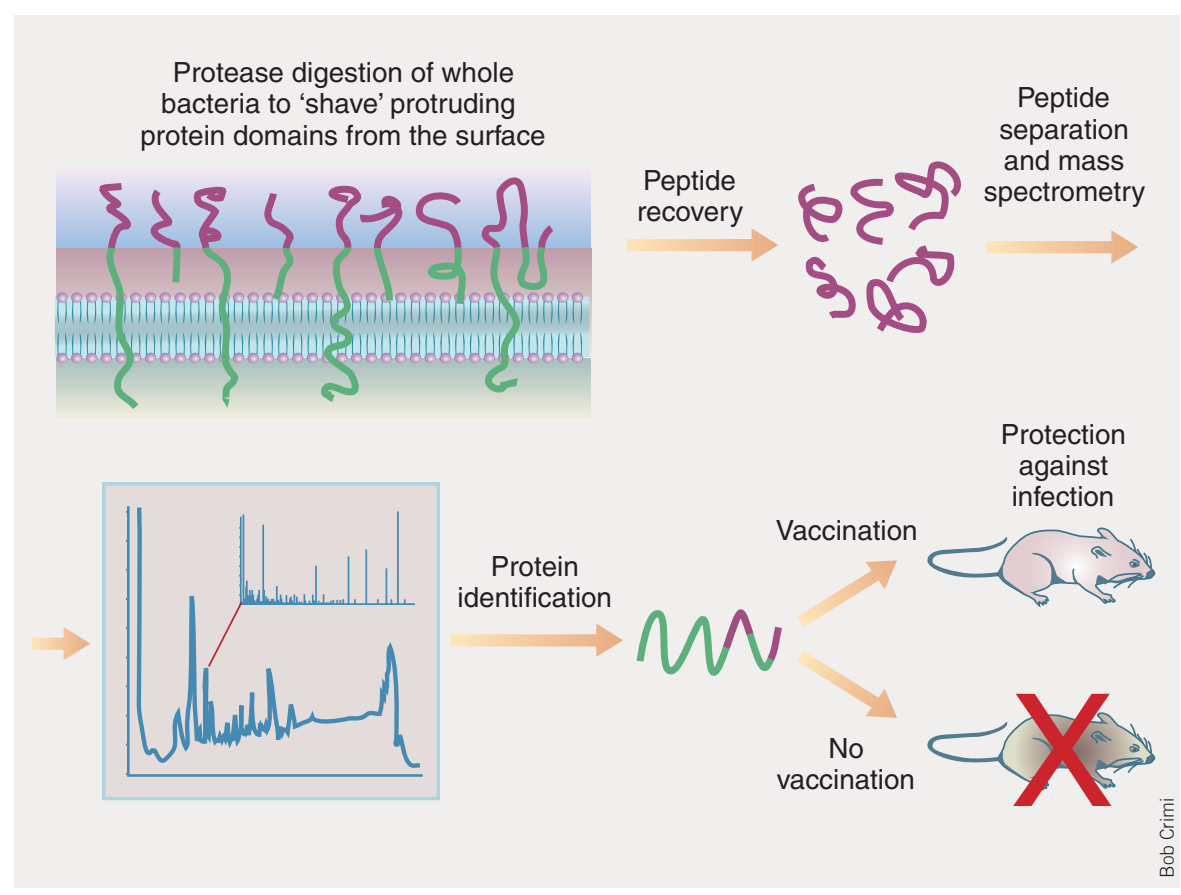

Figure 1 Strategy used to identify a new mouse-protective group A Streptococcus antigen. Bacteria are treated with trypsin to release surface-exposed peptides, which are subsequently identified by mass spectrometry. Genes encoding these proteins are cloned, and the overexpressed proteins are purified and used to immunize mice. The immunized animals are challenged with virulent group A Streptococcus to test for protection. 
treating group A Streptococcus with proteolytic enzymes such as trypsin destroyed the M protein antigen and thus altered the organism's antigenic composition.

Taking their cue from Lancefield's studies, Rodriguez-Ortega et al. treated serotype M1 group A Streptococcus strain SF370 with either trypsin or proteinase $\mathrm{K}$, resulting in release of cell-surface protein fragments. Subsequently, these fragments were concentrated, analyzed by tandem mass spectrometry and identified using bioinformatics interrogation of the publicly available genome sequence of this strain. Seventy-two proteins were identified, including 43 proteins deduced from trypsin-cleaved peptides, 18 from proteinase K-released peptides and 11 proteins from peptides obtained from both proteases. Importantly, only 4 of the 72 proteins were predicted by PSORT to be cytoplasmic proteins, which means that the technique was highly specific for secreted and/ or surface-associated proteins. These results were confirmed by fluorescence-activated cell sorting analysis using mouse polyclonal antibodies specific for 51 of the 72 identified proteins.

But the real beauty of this paper lies in the fact that the investigators did not rest on their technical accomplishments. Rather, they asked the logical next question: is there gold in the ore mined from cell-surface proteins? Based on the observation that 7 of the 11 previously reported group A Streptococcus protective antigens were identified successfully by their analysis, they tested the hypothesis that additional protective antigens were present among the 72 proteins identified. To do this, they first needed to define the cell-surface proteins of serotype M23 strain DSM2071, an organism (unlike strain SF370) that is highly virulent in mice. Seventeen proteins were identified in strain DSM2071, all of which have a homolog in strain SF370.

Turning next to the tried-and-true reverse vaccinology strategy pioneered by Chiron, 14 of the 17 proteins were overexpressed in recombinant form and used to immunize mice. Intranasal challenge of the immunized mice with strain DSM2071 revealed that two of the proteins conferred immunity in this model: $\mathrm{M}$ protein and a putative cell-envelope proteinase with a typical LPXTG cell-surface anchor motif. $\mathrm{M}$ protein has been known for decades to be a protective antigen ${ }^{6}$, whereas the protective ability of the putative proteinase had not been previously described.

Lest we be accused of unbridled enthusiasm, let us note some limitations of the study. First, the analysis was conducted with group A Streptococcus grown in vitro in a rich medium and harvested at a single growth phase. Inasmuch as group A Streptococcus is known to significantly remodel its transcriptome and proteome under different growth conditions (including in vitro versus in vivo) and at different growth phases ${ }^{8}$, the data presented are undoubtedly only a chapter rather than the whole book describing group A Streptococcus cell-surface proteins. Second, much of the work was done with strain SF370, an organism now known to be genetically divergent from the major M1 subclone responsible for the vast majority of contemporary infections ${ }^{9}$. Third, the authors identified only a relatively small proportion of the total number of proteins predicted by bioinformatics methods to be cell-anchored, lipoprotein, transmembranespanning and secreted. Especially noteworthy was the identification of only $6.5 \%$ of predicted transmembrane proteins. These issues should not be viewed as major flaws; rather, they are stimuli to conduct more extensive experiments that may produce additional discoveries.

What should be done next? From a broad perspective, clearly the strategy of Rodriguez-Ortega et al. can and should be applied to many other human and veterinary bacterial pathogens for which we lack efficacious vaccines. For example, Staphylococcus aureus continues to cause widespread human and animal disease. Undoubtedly, the strategy described by Rodriguez-Ortega et al. will be adopted rapidly by many investigators, and new vaccine candidates will be identified.

Several antigens have been reported to protect mice from experimental challenge with group A Streptococcus, generally in models of invasive infection ${ }^{10}$. It is recognized that a successful group A Streptococcus vaccine must be able to protect humans from pharyngotonsillitis caused by an extensive array of genetically diverse strains. Inasmuch as the cynomolgus macaque is the best animal model of this disease ${ }^{11}$, it will be important to test candidate antigens for their ability to protect monkeys from pharyngotonsillitis. Once that important milestone has been achieved and appropriate safety tests have been conducted, it will be imperative to move vaccine candidates rapidly to clinical testing.

1. Scarselli, M. et al. Trends Biotech. 23, 84-91 (2005).

2. Rodriguez-Ortega, M.J. et al. Nat. Biotechnol. 24, 191197 (2006)

3. Pizza, M. et al. Science 287, 1816-1820 (2000).

4. Maione, D. et al. Science 309, 148-150 (2005).

5. Carapetis, J.R. Lancet Infect. Dis. 5, 685-694 (2005).

6. Lancefield, R.C. Harvey Lectures, 36, 251 (19401941).

7. Lancefield, R.C. J. Exp. Med. 78, 465-476 (1943).

8. Musser, J.M. \& DeLeo, F. Am. J. Pathol. 167, 14611472 (2005).

9. Sumby, P. et al. J. Infect. Dis. 192, 771-782 (2005).

10. Bisno, A.L. et al. Clin. Infect. Dis. 41, 1150-1156 (2005).

11. Virtaneva, K. et al. Proc. Natl. Acad. Sci. USA 102 9014-9019 (2005).

\section{AAV does the shuffle}

\section{Aravind Asokan \& R Jude Samulski \\ Directed evolution of adeno-associated virus (AAV) yields mutant vectors that can evade neutralizing antibodies.}

DNA shuffling and related recombination techniques can dramatically accelerate the evolution of genetic mutations and enhance phenotypic traits. Such technology has been exploited to evolve proteins with improved stability and processing yields, enzymes with novel catalytic features, vaccines with increased immunogenicity and hybrid retroviruses ${ }^{1,2}$. In this issue, Maheshri et al. ${ }^{3}$ extend errorprone PCR and a staggered extension process (analogous to DNA shuffling) into the realm of recombinant AAV vectors. The study not

Aravind Asokan and R. Jude Samulski are at the Gene Therapy Center, 7113 Thurston Building, University of North Carolina at Chapel Hill, Chapel Hill, North Carolina 27599, USA.

e-mail: rjs@med.unc.edu only adds to a rapidly growing vector tool kit but also sheds light on structural aspects of the AAV capsid that may inspire strategies for the rational design of novel AAV vectors.

Recombinant AAV vectors are rapidly becoming the reagents of choice for therapeutic gene transfer. To date, eleven different serotypes, dubbed AAV1-11, and over 100 variants have been isolated from human or nonhuman primate tissues ${ }^{4}$. After the establishment of the first infectious clone of AAV2 in 1982, recombinant AAV2 (rAAV2) vectors rapidly gained popularity in gene therapy applications owing to their lack of pathogenicity, expanded tropism and ability to establish long-term transgene expression. Recombinant AAV2 vectors have been tested in preclinical studies for the treatment of hemophilia, alpha-1 anti-trypsin deficiency, cystic fibrosis, Duchenne muscular 